\title{
The SARS-CoV-2 host cell membrane fusion protein TMPRSS2 is a tumor suppressor and its downregulation promotes antitumor immunity and immunotherapy response in lung adenocarcinoma
}

Xiaosheng Wang ( $\nabla$ xiaosheng.wang@cpu.edu.cn )

China Pharmaceutical University https://orcid.org/0000-0002-7199-7093

Zhixian Liu

Jiangsu Cancer Hospital

Zhilan Zhang

China Pharmaceutical University

Qiushi Feng

China Pharmaceutical University

\section{Research Article}

Keywords: lung adenocarcinoma, SARS-CoV-2, TMPRSS2, antitumor immune response, tumor progression

Posted Date: February 22nd, 2022

DOI: https://doi.org/10.21203/rs.3.rs-740479/v3

License: (c) (i) This work is licensed under a Creative Commons Attribution 4.0 International License.

Read Full License 


\section{Abstract}

\section{Background}

TMPRSS2, a key molecule for SARS-CoV-2 invading human host cells, has an association with cancer. However, its association with lung cancer remains unexplored.

\section{Results}

In five lung adenocarcinoma (LUAD) genomics datasets, we explored associations between TMPRSS2 expression and immune signatures, tumor progression phenotypes, and clinical prognosis in LUAD by the bioinformatics approach. We found that TMPRSS2 expression levels correlated negatively with the enrichment levels of both immune-stimulatory and immune-inhibitory signatures, while they correlated positively with the ratios of immune-stimulatory/immune-inhibitory signatures. It indicated that TMPRSS2 levels had a stronger negative correlation with immune-inhibitory than with immune-stimulatory signatures. TMPRSS2 downregulation correlated with increased proliferation, stemness, genomic instability, tumor progression, and worse survival in LUAD. We further validated that TMPRSS2 was downregulated with tumor progression in the LUAD dataset we collected. In vitro and in vivo experiments verified the association of TMPRSS2 deficiency with increased tumor cell proliferation and invasion and antitumor immunity in LUAD. Moreover, in vivo experiments demonstrated that TMPRSS2-knockdown tumors were more sensitive to BMS-1, an inhibitor of PD-1/PD-L1.

\section{Conclusions}

TMPRSS2 is a tumor suppressor, while its downregulation is a positive biomarker of immunotherapy in $\angle U A D$. Our data provide a link between lung cancer and pneumonia caused by SARS-CoV- 2 infection .

\section{Introduction}

The severe acute respiratory syndrome coronavirus 2 (SARS-CoV-2) has infected more than 204 million people and caused more than 4.3 million deaths worldwide as of August 12, 2021

(https://coronavirus.jhu.edu/map.html). SARS-CoV-2 invades host cells using its spike glycoprotein (S), ${ }^{1}$ which is composed of S1 and S2 functional domains. S1 binds the angiotensin-converting enzyme 2 (ACE2) for cell attachment, and S2 binds the transmembrane protease serine 2 (TMPRSS2) for membrane fusion. ${ }^{1}$ Since TMPRSS 2 plays a crucial role in the regulation of SARS-CoV- 2 invasion, and cancer patients are susceptible to SARS-CoV-2 infection, an investigation into the role of TMPRSS2 in cancer is significant in the context of the current SARS-CoV-2 pandemic. Previous studies have demonstrated the association between TMPRSS2 and cancer. ${ }^{2-5}$ Typically, the TMPRSS2-ERG gene fusion frequently occurs in prostate cancer and is associated with tumor progression. ${ }^{6-8}$ In a recent study, ${ }^{3}$ Katopodis et al. revealed that TMPRSS2 was overexpressed in various cancers versus their normal tissues. In another study, ${ }^{4}$ Kong et al. explored TMPRSS2 expression in lung adenocarcinoma (LUAD) and lung squamous cell carcinoma (LUSC). This study suggested that TMPRSS2 was a tumor suppresser in 
LUAD for its significant downregulation in LUAD versus normal tissue. A few studies have examined the association between TMPRSS 2 and tumor immunity in cancer. For example, Bao et al. ${ }^{5}$ investigated TMPRSS2 expression and its associations with immune and microbiome variates across 33 tumor types. Luo et al. ${ }^{9}$ explored the association between TMPRSS2 expression and immune infiltration in prostate cancer. Despite these prior studies, the associations of TMPRSS2 with tumor immunity, oncogenic signatures or pathways, tumor progression and clinical outcomes in lung cancer remain insufficiently explored.

In this study, we analyzed the associations between TMPRSS2 expression levels and the enrichment levels of immune signatures in five LUAD cohorts. The immune signatures included CD8+ T cells, NK cells, immune cytolytic activity, CD4+ regulatory T cells, myeloid-derived suppressor cells (MDSCs), and PD-L1. We also analyzed the associations between TMPRSS2 expression levels and the activities of several oncogenic pathways, including cell cycle, mismatch repair, and p53 signaling. Moreover, we explored the associations between TMPRSS2 expression and tumor phenotypes (such as proliferation and tumor stemness), genomic features (such as genomic instability and intratumor heterogeneity (ITH)), tumor advancement and prognosis in these LUAD cohorts. Furthermore, we explored the association between TMPRSS2 expression and the response to cancer immunotherapy. We validated the computational findings by performing in vitro experiments in the human lung cancer cell line A549 and in vivo experiments with mouse tumor models. We also validated our findings in LUAD patients we collected from Jiangsu Cancer Hospital, China. Our study demonstrates that TMPRSS2 is a tumor suppressor while its downregulation can promote antitumor immune response and cancer immunotherapy response. This study may provide insights into the connection between lung cancer and pneumonia caused by SARS-CoV-2 infection.

\section{Materials And Methods}

\section{Datasets}

We downloaded RNA-Seq gene expression profiling (level 3 and RSEM normalized), protein expression profiling, and clinical data for the TCGA-LUAD cohort from the Genomic Data Commons Data Portal (https://portal.gdc.cancer.gov/). We downloaded microarray gene expression profiling (normalized) and clinical data for other four LUAD cohorts (GSE12667, ${ }^{10}$ GSE30219, ${ }^{11}$ GSE31210, ${ }^{12}$ and GSE50081 ${ }^{13}$ ) from the Gene Expression Omnibus (https://www.ncbi.nlm.nih.gov/geo/). In addition, we collected 100 blood samples from LUAD patients and 20 blood samples from healthy persons from Jiangsu Cancer Hospital, China. According to the diagnosis and treatment guidelines for non-small cell lung cancer (CSCO 2020), LUAD patients in this study were divided into two groups: 50 patients in early stage (stage I) and 50 patients in late stage (stage III-IV). We log2-transformed the RNA-Seq gene expression values before further analyses. A description of these datasets is shown in Supplementary Table S1.

\section{Gene-set enrichment analysis}


We quantified the enrichment levels of immune signatures, pathways, and tumor phenotypes in tumors by the single-sample gene-set enrichment analysis (SSGSEA) ${ }^{14}$ of their marker gene sets. The ssGSEA was performed with the R package "GSVA". ${ }^{14}$ The marker gene sets are presented in Supplementary Table S2. We used GSEA ${ }^{15}$ to identify KEGG ${ }^{16}$ pathways significantly associated with a gene set with a threshold of adjusted $p$ value $<0.05$. We used WGCNA, ${ }^{17}$ an R package, to identify gene modules and their associated gene ontology (GO) terms enriched in the high- (upper third) and low-TMPRSS2expression-level (bottom third) LUADs.

\section{Survival Analysis}

We compared overall survival (OS) and disease-free survival (DFS) between the high- (upper third) and low-TMPRSS2-expression-level (bottom third) LUAD patients. Kaplan-Meier curves were utilized to display survival time differences, whose significances were evaluated by the log-rank test. We performed the survival analyses using the R package "survival".

\section{Statistical analysis}

We used the Spearman correlation to evaluate associations between TMPRSS2 expression levels and sSGSEA scores of gene sets; the Spearman correlation coefficients $(\rho)$ and $p$ values were reported. In addition, we used the Pearson correlation to evaluate associations between TMPRSS2 expression levels and gene or protein expression levels and the ratios of immune signatures; the Pearson correlation coefficients $(r)$ were reported. The ratios between immune signatures were the log2-transformed values of the ratios between the geometric mean expression levels of all marker genes in immune signatures. In comparisons of TMPRSS2 expression levels between two classes of samples, we used the two-tailed Student's $t$ test. We performed the statistical analyses using the R programming software (https://cran.rproject.org/).

\section{In vitro experiments}

\section{Antibodies, reagents and cell lines}

All antibodies were used at a dilution of 1:1000 unless otherwise specified. Anti-PD- L1 (ab213480), antiCD8 (ab22378), anti-CD49b (ab181548), anti-MSH6 (ab92471), anti-TMPRSS2 (ab109131) and antiGAPDH (ab181603) were purchased from Abcam (Burlingame, CA). PE anti-mouse TNF-a antibody (127321-81), APC anti-mouse IFN-y antibody (17-7311-81), APC anti-mouse CD279 (PD-1) antibody (129985-81), and APC anti-mouse CD223 (LAG-3) antibody (12-2231-81) were purchased from eBioscience (San Diego, CA). The human lung cancer cell lines A549 were from the American Type Culture Collection. They were cultured in $90 \%$ F12K (GIBCO, USA) supplemented with $10 \%$ fetal bovine serum in a humidified 
incubator at $37^{\circ} \mathrm{C}$ and 5\% CO2. NK92 cells (KeyGEN BioTECH, Nanjing, China) were cultured in Alpha MEM (GIBCO, USA) with $2 \mathrm{mM}$ L-glutamine, $1.5 \mathrm{~g} / \mathrm{L}$ sodium bicarbonate, $0.2 \mathrm{mM}$ inositol, $0.1 \mathrm{mM} 2-$ mercaptoethanol, 0.02 mM folic acid, 100-200 U/mL recombinant human IL-2 (PeproTech, Rocky Hill, New Jersey, USA), and a final concentration of $12.5 \%$ horse serum and $12.5 \%$ fetal bovine serum.

\section{TMPRSS2 knockdown with small interfering RNA (siRNA)}

A549 cells were transfected with TMPRSS2 siRNA or control siRNA by using Effectene Transfection Reagent (Qiagen, Hilden, Germany, B00118) according to the manufacturer's instructions. The medium was replaced after 24 hours incubation with fresh medium, and the cells were maintained for a further 24 hours. Quantitative PCR or Western blotting were used to detect the transfection efficiency. TMPRSS2 SiRNA and control siRNA were synthesized by KeyGEN Biotech (Nanjing, China). Their sequences were as follows: TMPRSS2 siRNA: 1, 5'- GGAC AUGG GCUA UAAG AAU -3' (sense) and 5'- AUUC UUAU AGCC CAUG UCC-3' (antisense); 2, 5'- ACUC CAAG ACCA AGAA CAA -3' (sense) and 5'- UUGU UCUU GGUC UUGG AGU-3' (antisense); 3,5'-GGAC UGGA UUUA UCGA CAA-3'(sense) and 5'-UUGU CGAU AAAU CCAG UCC-3' (antisense); control siRNA: 5'-UUCU CCGA ACGU GUCA CGU dTdT-3' (sense) and 5'ACGUGACACGUUCGGAGAAdTdT-3' (antisense).

\section{Lentivirus generation and infection}

Lentivirus was prepared according to the manufacturer's instructions. The heteroduplexes, supplied as 58-nucleotide oligomers, were annealed; the downstream of the U6 promoter was inserted into the pLKO.1 plasmid to generate pLKO.1/ShTMPRSS2. Recombinant and control lentiviruses were produced by transiently transfecting pLK0.1/vector and pLKO.1/ShTMPRSS2, respectively. The lentiviruses were transfected into 293 T cells. After 48 hours, lentiviral particles were collected and concentrated from the supernatant by ultracentrifugation. Effective lentiviral shRNA was screened by infecting these viruses with Lewis cells, and their inhibitory effect on TMPRSS2 expression was analyzed by quantitative PCR and Western blotting. The lentivirus containing the ShTMPRSS2 RNA target sequences and a control virus were used for the animal study. The coding strand sequence of the shRNA-encoding oligonucleotides was 5'-ACGGGAACGTGACGGTATTTA-3' for TMPRSS2.

\section{Western blotting}

A549 cell extracts were lysed by using lysis buffer supplemented with protease inhibitor cocktail immediately before use. Total proteins present in the cell lysates were quantified by using the BCA assay. Proteins were denatured by addition of 6 volumes of SDS sample buffer and boiled at $95^{\circ} \mathrm{C}$ for 5 min and were then separated by SDS-PAGE. The resolved proteins were transferred onto a nitrocellulose membrane after electrophoresis. The membranes were incubated with $5 \%$ skimmed milk in TBS containing $0.1 \%$ Tween 20 (TBS-T) for 1 hour to block the non-specific binding and then incubated 
overnight at $4^{\circ} \mathrm{C}$ with specific antibodies. After 2 hours incubation with the HRP-labeled secondary antibody, proteins were visualized by enhanced chemiluminescence using a G: BOX chemiXR5 digital imaging system (SYNGENE, UK). The band densities were normalized to the background, and the relative optical density ratios were calculated relative to the housekeeping gene GAPDH.

\section{Quantitative PCR}

The total RNA was isolated by Trizol (Invitrogen, USA) and was reversely transcribed into cDNA using the RevertAid First Strand cDNA Synthesis Kit (Thermo Fisher, USA). Quantitative PCR was performed with the ABI Step one plus Real-Time PCR (RT-PCR) system (ABI, USA) using One Step TB Green ${ }^{\text {TM }}$ PrimeScript ${ }^{\text {TM }}$ RT-PCR Kit II (SYBR Green) (RR086B, TaKaRa, JAPAN). Relative copy number was determined by calculating the fold-change difference in the gene of interest relative to GAPTH. The program for amplification was one cycle of $95^{\circ} \mathrm{C}$ for $5 \mathrm{~min}$, followed by 40 cycles of $95^{\circ} \mathrm{C}$ for $15 \mathrm{sec}, 60^{\circ} \mathrm{C}$ for $20 \mathrm{sec}$, and $72^{\circ} \mathrm{C}$ for $40 \mathrm{sec}$. The relative amount of each gene was normalized to the amount of GAPDH. The primer sequences were as follows: hTMPRSS2: 5'-AACT TCAT CCTT CAGG TGTA-3' (forward) and 5'TCTC GTTC CAGT CGTCTT-3' (reverse); hGAPDH: 5'- AGAT CATC AGCA ATGC CTCCT-3' (forward) and 5'ACAC CATG TATT CCGG GTCAAT-3' (reverse).

\section{Cell proliferation assay}

A549 cells were plated in 96 -well plates at $3 \times 10^{4}$ cells per well and maintained in a medium containing 10\% FBS. After 24 hours, cell proliferation was determined using the Cell Counting Kit-8 (CCK-8; KeyGEN Biotech, China) following the manufacturer's instructions. To perform the CCK-8 assay, $10 \mu$ CCK-8 reagent was added to each well and the 96 plates were incubated at $37^{\circ} \mathrm{C}$ for 2 hours. The optical density was read at $450 \mathrm{~nm}$ using a microplate reader. All these experiments were performed in triplicates.

\section{Transwell migration and invasion assays}

Cell migratory and invasive abilities were assessed using 24 well transwell chambers (Corning, USA) with membrane pore size of $8.0 \mu \mathrm{m}$. A549 cells were seeded into the upper chamber without matrigel at $1 \times 10^{5}$ cells in serum-free medium, while $500 \mu \mathrm{l}$ medium containing $20 \%$ FBS was added to the lower chamber. The chambers were incubated at $37^{\circ} \mathrm{C}$ and $5 \% \mathrm{CO}_{2}$ for 24 hours. The cells on the upper chamber were scraped off with cotton-tipped swabs, and cells that had migrated through the membrane were stained with $0.1 \%$ crystal violet at $37^{\circ} \mathrm{C}$ for $30 \mathrm{~min}$. The migrated cells were counted at $200 \mathrm{x}$ magnification under the microscope using three randomly selected visual fields. All these experiments were performed in triplicates.

\section{Co-culture of tumor cells with NK92 cells}


A transwell chamber (Corning, USA) was inserted into a six well plate to construct a co-culture system. A549 cells were seeded on the six well plate at a density of $5 \times 10^{4}$ cells/well, and NK92 cells were seeded on the membrane (polyethylene terephthalate, pore size of $0.4 \mu \mathrm{m}$ ) of the transwell chamber at a density of $5 \times 10^{4}$ cells/chamber. Tumor cells and NK92 cells were co-cultured in a humidified incubator at $37^{\circ} \mathrm{C}$ and $5 \% \mathrm{CO}_{2}$ atmosphere for 48 hours.

\section{EdU proliferation assay}

After co-culture of A549 cells with NK92 cells for 48 hours, we measured the proliferation capacity of NK92 cells by an EdU (5- ethynyl-2'-deoxyuridine; Invi-trogen, California, USA) proliferation assay. NK92 cells were plated in 96 -well plates with a density of $2 \times 10^{3}$ cells/well with $10 \mu \mathrm{M} \mathrm{EdU} \mathrm{at} 37^{\circ} \mathrm{C}$ for 24 hours. The cell nuclei were stained with 4',6- diamidino-2-phenylindole (DAPI) at a concentration of $1 \mu \mathrm{g} / \mathrm{mL}$ for 20 min. The proportion of NK92 cells incorporating EdU was detected with fluorescence microscopy. All the experiments were performed in triplicates.

\section{In vivo experiments}

\section{In vivo mouse models}

Lewis tumor cells were transduced with ShCon (scramble) or ShTMPRSS2 lentivirus and selected by puromycin for 7 days. The stably transfected Lewis tumor cells $(1 \times 107 / \mathrm{ml})$ were subcutaneously injected into the right armpit of recipient mice after shaving the injection site. After 5 days, when the tumor volume was approximately $4-5 \mathrm{~mm} 3$, the mice were randomly divided into six groups, with half of the ShCon and ShTMPRSS2 mice treated with $150 \mathrm{U} / \mathrm{L}$ PD1/PDL1 inhibitor BMS-1 (concentration $500 \mathrm{mg} / \mathrm{mL}$; i.p.) (MCE Cat. No. HY-19991) every 3 days. The tumors were isolated from mice after 15 days. Tumor volumes did not exceed the maximum allowable size according to the LJI IACUC animal experimental protocol. The tumor volume was measured every 3 days after the tumor appeared on the fifth day and was calculated as follows: $\mathrm{V}=1 / 2 \times$ width $2 \times$ length.

\section{Isolation of tumor-infiltrating lymphocytes (TILs)}

After the tumor tissues were separated aseptically and rinsed with cold PBS for 3 times, they were excised and chopped with tweezers and scissors and were then digested with $2 \mathrm{mg} / \mathrm{mL}$ collagenase (type IV, sigma V900893) for 45 min, until no tissue mass was visible. Following digestion, lymphocytes were separated with lymphocyte separation medium, washed with PBS, and counted. The specific protocol was as follows: tumors were filtered through $70 \mu \mathrm{M}$ cell strainers, and the cell suspension was washed twice in culture medium by centrifugation at $1500 \mathrm{rpm}$ and $4^{\circ} \mathrm{C}$ for $10 \mathrm{~min}$. After the washing, the cells were resuspended with PBS and were layered over $3 \mathrm{~mL}$ of 30\%-100\% gradient percoll (Beijing Solarbio 
Science \& Technology, Beijing, China); this was followed by centrifugation at $2600 \mathrm{rpm}$ for $25 \mathrm{~min}$ at $25^{\circ} \mathrm{C}$. The enriched TILs were obtained at the interface as a thin buffy layer, were washed with PBS three times, and finally were resuspended in FACS staining buffer for further staining procedures.

\section{Flow cytometry}

TILs were stained with CD8 (eBioscience, 11-0081-81), CD49b (eBioscience, 11-5971-81), PD-1 (eBioscience, 12-9985-81), and LAG3 (eBioscience, 12-2231-81) and were analyzed by flow cytometry. TILs were restimulated with cell stimulation cocktail (eBioscience, San Diego, California, USA), and the expression of IFN-y and TNF-a (Biolegend) was analyzed by flow cytometry. Staining for cell surface markers was performed by incubating cells with antibody (1:100 dilution) in FACS buffer ( $0.1 \%$ BSA in PBS) for $30 \mathrm{~min}$ at $4^{\circ} \mathrm{C}$. Surface markers of intracellular cytokines (IFN-y (eBioscience, 17-7311-81) and TNF-a (eBioscience, 12-7321-81)) were stained before fixation/permeabi-lization (Intracellular Fixation \& Permeabilization Buffer Set, ThermoFisher).

\section{Immunofluorescence of CD8, CD49b and PD-L1}

Paraffin-embedded mice tumor tissue sections ( $3 \mu \mathrm{m}$ thick) were subjected to immunofluorescence with CD8 (Abcam, ab22378), CD49b (Abcam, ab181548), or PD-L1 (Abcam, ab2134808) primary antibodies. Before immunostaining, tumor tissue sections were deparaffinized with xylene, rehydrated and unmasked in sodium citrate buffer $(10 \mathrm{mM}, \mathrm{pH} 6.0)$, and treated with a glycine solution $(2 \mathrm{mg} / \mathrm{mL})$ to quench autofluorescence. After antigen retrieval, 3\% H2O2-methanol solution blocking inactivated enzymes, and goat serum blocking, tissue slides were incubated in wet box for 2 hours at $37^{\circ} \mathrm{C}$ with anti-CD8, CD $49 \mathrm{~b}$, or anti-PD-L1 rabbit primary antibodies (1:100 dilution) in blocking solution, and were then dropped with FITC (1:100 dilution) secondary antibody 50-100ul and incubated at $37^{\circ}$ for 1 hour in the dark. The immunolabeled slides were examined with a fluorescence microscope after nuclear counterstaining with DAPI. Green, red and blue channel fluorescence images were acquired with a Leica DFC310 FX 1.4megapixel digital color camera equipped with LAS V.3.8 software (Leica Microsystems, Wetzlar, Germany). Overlay images were reconstructed by using the free-share ImageJ software.

\section{Results}

\section{Associations between TMPRSS2 expression and immune signatures in LUAD}

We found that TMPRSS2 had a significant negative expression correlation with the infiltration levels of CD8+ T cells, which represent the adaptive antitumor immune response, in three of the five LUAD cohorts (Spearman correlation, $p<0.05$ ) (Figure 1a). TMPRSS2 expression levels were also significantly and negatively correlated with the infiltration levels of NK cells, which represent the innate antitumor immune 
response, in two LUAD cohorts $(p<0.05)$ (Figure 1a). Moreover, TMPRSS2 expression levels were negatively correlated with immune cytolytic activity, a marker for underlying immunity, ${ }^{18}$ in all the five LUAD cohorts. Meanwhile, TMPRSS 2 had a significant negative expression correlation with $P D-L 1$ in the five LUAD cohorts (Figure 1a). TMPRSS2 expression levels were negatively correlated with the infiltration levels of CD4+ regulatory T cells and MDSCs in four LUAD cohorts, which represent tumor immunosuppressive signatures (Figure 1a). Taken together, these results suggest a significant negative association between TMPRSS2 abundance and immune infiltration levels in LUAD. Interestingly, TMPRSS2 expression levels showed a significant positive correlation with the ratios of immunestimulatory/immune-inhibitory signatures (CD8+ T cells/PD-L1) consistently in the five LUAD cohorts (Pearson correlation, $p<0.05$ ) (Figure 1b). It indicated that TMPRSS2 levels had a stronger negative correlation with immune-inhibitory signatures than with immune-stimulatory signatures. Furthermore, we found that the ratios of immune-stimulatory/immune-inhibitory signatures were positively correlated with DFS in the TCGA-LUAD cohort (log-rank test, $p=0.01$ ) (Figure 1c).

\section{Associations between TMPRSS2 expression and oncogenic pathways, tumor phenotypes and prognosis in LUAD}

We found that TMPRSS2 expression levels were inversely correlated with the activities of the cell cycle, mismatch repair, and p53 signaling pathways in the five LUAD cohorts (Spearman correlation, $p<0.001$ ) (Figure 2a). Moreover, TMPRSS2 showed a negative expression correlation with MKI67, a tumor proliferation marker, in the five LUAD cohorts (Pearson correlation, $p<0.001$ ) (Figure 2b). Tumor stemness indicates a stem cell-like tumor phenotype representing an unfavorable prognosis in cancer. ${ }^{19}$ We observed that TMPRSS2 expression levels were inversely correlated with tumor stemness scores in these LUAD cohorts (Spearman correlation, $p<0.001$ ) (Figure 2c).

We detected that TMPRSS2 expression levels significantly decreased with tumor advancement in LUAD (Figure 2d). For example, in the TCGA-LUAD cohort, TMPRSS2 expression levels were significantly lower in late-stage (Stage III-IV) than in early-stage (Stage I-II) LUADs (Student's $t$ test, $p<0.001$; fold change $(\mathrm{FC})=1.6)$, in large-size (T3-4) than in small-size (T1-2) LUADs $(p=0.007 ; \mathrm{FC}=1.5)$, in LUADs with lymph nodes (N1-3) than in those without regional lymph nodes (N0) $(p=0.02 ; \mathrm{FC}=1.3)$, and in LUADs with metastasis (M1) than in those without metastasis (M0) $(p=0.07 ; \mathrm{FC}=1.6)$. In other two LUAD cohorts (GSE30219 and GSE50081) with tumor size and lymph nodes data available, TMPRSS2 expression levels were also significantly lower in large-size than in small-size LUADs $(p<0.001 ; F C=6.4)$ in GSE30219 and were significantly lower in N1-3 than in N0 LUADs in both GSE30219 $(p=0.02 ; \mathrm{FC}=2.83)$ and GSE50081 $(p=0.02 ; \mathrm{FC}=1.6)$ (Figure 2d). Furthermore, the lung cancer data from Jiangsu Cancer Hospital supported that TMPRSS2 expression levels were reduced in late-stage (Stage IV) than in early-stage (Stage I-II) LUADs $(p<0.001 ; F C=1.6)$ (Figure 2e). Survival analyses showed that TMPRSS2 downregulation was correlated with worse OS and/or DFS in these LUAD cohorts (log-rank test, $p<0.05$ ) (Figure 2f). 
It has been shown that EGFR-mutated LUADs have a better prognosis than EGFR-wildtype LUADs. ${ }^{20}$ We found that TMPRSS2 was more lowly expressed in EGFR-wildtype than in EGFR-mutated LUADs ( $p=$ $0.006 ; F C=1.5)$ (Figure 2g). Besides, LUAD harbors three transcriptional subtypes: terminal respiratory unit (TRU), proximal-inflammatory (PI), and proximal-proliferative (PP), of which TRU has the best

prognosis. ${ }^{21}$ We found that TMPRSS2 expression levels were the highest in TRU (TRU versus PP: $p=8.68$ $\times 10^{-14}, \mathrm{FC}=2.98$; TRU versus PI: $p=1.07 \times 10^{-11}, \mathrm{FC}=3.16$ ) (Figure $2 \mathrm{~g}$ ).

Taken together, these results suggest that TMPRSS2 downregulation is associated with worse outcomes in LUAD.

\section{Association between TMPRSS2 expression and genomic instability in LUAD}

Genomic instability plays prominent roles in cancer initiation, progression, and immune invasion ${ }^{22}$ by increasing tumor mutation burden $(\mathrm{TMB})^{23}$ and aneuploidy or somatic copy number alterations. ${ }^{24}$ In the TCGA-LUAD cohort, TMPRSS2 expression levels had a negative correlation with TMB (Spearman correlation, $\rho=-0.31 ; p=2.58 \times 10^{-12}$ ) (Figure 3a). Homologous recombination deficiency (HRD) may promote chromosomal instability and aneuploidy levels in cancer. ${ }^{25}$ We found that TMPRSS2 expression levels were inversely correlated with HRD scores ${ }^{25}$ in $\operatorname{LUAD}\left(\rho=-0.27 ; p=5.76 \times 10^{-10}\right)$ (Figure 3b). DNA damage repair (DDR) deficiency can lead to genomic instability. ${ }^{26}$ Knijnenburg et al. ${ }^{25}$ identified deleterious gene mutations for nine DDR pathways in TCGA cancers. We divided LUAD into pathwaywildtype and pathway-mutated subtypes for each of the nine DDR pathways. The pathway-wildtype indicates no deleterious mutations in any pathway genes, and the pathway-mutated indicates at least a deleterious mutation in pathway genes. Interestingly, we found that TMPRSS2 expression levels were significantly lower in the pathway-mutated subtype than in the pathway-wildtype subtype for seven DDR pathways ( $p<0.05 ; \mathrm{FC}>1.5$ ) (Figure $3 c$ ). The seven pathways included base excision repair, Fanconi anemia, homologous recombination, mismatch repair, nucleotide excision repair, translesion DNA synthesis, and damage sensor. These results suggest a correlation between TMPRSS2 downregulation and DDR deficiency.

TP53 mutations often leads to genomic instability because of the important role of p53 in maintaining genomic stability. ${ }^{27}$ We found that TMPRSS2 displayed significantly lower expression levels in TP53mutated than in TP53-wildtype LUADs ( $p=0.006 ; \mathrm{FC}=1.5$ ) (Figure 3d). Moreover, we found numerous DDR-associated genes having significant negative expression correlations with TMPRSS2 in these LUAD cohorts (Pearson correlation, $p<0.05$ ), including MSH2, MSH6, POLE, PCNA, and RAD51 (Figure 3e). Furthermore, we observed significant negative expression correlations between TMPRSS2 and DNA mismatch repair proteins MSH6 (Pearson correlation, $\left.r=-0.30 ; p=6.6 \times 10^{-9}\right)$ and PCNA $(r=-0.25 ; p=1.5$ $\times 10^{-6}$ ) in the TCGA-LUAD cohort (Figure $3 f$ ). These results indicated an association between TMPRSS2 downregulation and the upregulation of DDR molecules, the signature of increased genomic instability. 
Genomic instability can promote tumor heterogeneity, which is associated with tumor progression, immune evasion, and drug resistance. ${ }^{28}$ We used the DEPTH algorithm ${ }^{29}$ to score ITH for each TCGALUAD sample and found a significant negative correlation between TMPRSS2 expression levels and ITH scores in LUAD ( $\rho=-0.55 ; p<0.001$ ) (Figure $3 g)$. It indicates a significant association between TMPRSS2 downregulation and increased ITH in LUAD.

Taken together, these results suggest that TMPRSS2 downregulation is associated with enhanced genomic instability in LUAD.

\section{Co-expression networks of TMPRSS2 in LUAD}

We found 150 and 135 genes having strong positive and negative expression correlations with TMPRSS2 in the TCGA-LUAD cohort, respectively (Pearson correlation, $|\eta|>0.5$ ) (Figure 4a; Supplementary Table S3). Gene set enrichment analysis (GSEA) ${ }^{14}$ revealed that the cell cycle, p53 signaling, mismatch repair, and homologous recombination pathways were significantly associated with the 135 genes with strong negative expression correlations with TMPRSS2. This conforms to the previous findings that TMPRSS2 downregulation was correlated with increased activities of these pathways.

Weighted gene co-expression network analysis (WGCNA) ${ }^{17}$ identified six gene modules (indicated in blue, turquoise, brown, magenta, purple, and pink color, respectively) highly enriched in the high-TMPRSS2expression-level LUADs. The representative GO terms associated with these modules included cell projection, chromosome segregation, response to endogenous stimulus, cell adhesion, cellular response to lipopolysaccharide, and micro-ribonucleoprotein complex. In contrast, three gene modules (indicated in green, black, and green-yellow color, respectively) were highly enriched in the low-TMPRSS2-expressionlevel LUADs (Figure 4b). The representative GO terms for these modules included extracellular matrix (ECM), small molecule metabolic process, and postsynapse (Figure 4b). The ECM signature plays a crucial role in driving cancer progression. ${ }^{30}$ Its upregulation in the low-TMPRSS2-expression-level LUADs is in accordance with the correlation between TMPRSS2 downregulation and LUAD progression.

\section{Experimental validation of the bioinformatics findings}

To validate the findings from the bioinformatics analysis, we performed in vitro experiments with the human LUAD cell line A549 and in vivo experiments with mouse tumor models. We found that TMPRSS2 knockdown markedly promoted proliferation and invasion potential in A549 cells (Figure 5a) and increased tumor volume and progression in Lewis tumor mouse models (Figure 5b). This is consistent with the previous results showing that TMPRSS2 downregulation is associated with tumor progression and unfavorable prognosis in LUAD. Furthermore, in vitro experiments showed that MSH6 expression was upregulated in TMPRSS2-knockdown versus TMPRSS2-wildtype A549 cells (Figure 5c). This is in line with the previous finding of the significant negative correlation between TMPRSS2 expression levels and MSH6 abundance in LUAD. 
Our bioinformatics analysis revealed a significant inverse correlation between TMPRSS2 abundance and immune infiltration levels in LUAD. Consistently, the MHC class I genes ( $H L A-A, H L A-B$, and $H L A-C)$ showed significantly higher expression levels in TMPRSS2-knockdown than in TMPRSS2-wildtype A549 cells, demonstrated by real-time qPCR (Figure 5d). NK cells co-cultured with TMPRSS2-knockdown A549 cells displayed significantly stronger proliferation ability than NK cells co-cultured with TMPRSS2wildtype A549 cells, evident by the EdU proliferation assay (Figure 5e). Furthermore, in vivo experiments showed that infiltration of CD8+ T cells and NK cells significantly increased in TMPRSS2-knockdown tumors (Figure 5f). Moreover, on CD8+ T cells from tumor-infiltrating lymphocytes (TILs) in TMPRSS2knockdown tumors, the expression of TNF-a and IFN-y were significantly upregulated (Figure 5g, h), indicating that TMPRSS2 knockdown can enhance the activity of CD8+ TILs. Meanwhile, the expression of PD-1 and LAG3 also significantly increased on CD8+ TILs in TMPRSS2-knockdown tumors (Figure 5i, j), indicating that TMPRSS2 deficiency can also promote the exhaustion of CD8+ TILs.

Our bioinformatics analysis revealed a significant negative correlation between TMPRSS2 and PD-L 1 expression levels. This result was confirmed by both in vitro and in vivo experiments; knockdown of TMPRSS2 increased PD-L1 expression in A549 cells, as evidenced by Western blotting (Figure 5c); TMPRSS2-knockdown tumors had significantly enhanced PD-L1 expression (Figure 5f). Furthermore, bioinformatics analysis revealed a significant positive correlation between TMPRSS2 expression levels and the ratios of CD8+ T cells/PD-L1. This was confirmed by that TMPRSS2-knockdown tumors displayed a higher level of increases in CD8+ T cell infiltration than in PD-L1 abundance (Figure 5f). Because PD-L1 expression is a predictive biomarker of response to immune checkpoint inhibitors (ICls) in cancer, $^{31}$ we anticipated that knockdown of TMPRSS2 would promote the response to ICIs in LUAD. As expected, the volume of the TMPRSS2-knockdown tumors had a significantly higher level of decreases than that of TMPRSS2-wildtype tumors after treatment with BMS-1, an inhibitor of PD-1/PD-L1 (Figure $5 \mathrm{k}$; this result supports that knockdown of TMPRSS2 can enhance the sensitivity of LUAD to the PD1/PD-L1 inhibitor. Furthermore, the activities of CD8+ TILs and NK TILs markedly increased in TMPRSS2knockdown tumors after treatment with BMS-1; they were significantly higher in TMPRSS2-knockdown than in TMPRSS2-wildtype tumors after treatment with BMS-1 (Figure 5l, $\mathrm{m}$ ). These results support that the PD-1/PD-L1 inhibitor promotes immune elimination of tumor cells by inhibiting the exhaustion of CD8+ TILs and NK TILs in TMPRSS2-depleted LUAD.

To summarize, bioinformatics analysis revealed a negative correlation between TMPRSS2 abundance and immune infiltration levels in LUAD. Experimental results demonstrated that this relationship was a causal relationship. That is, reduced TMPRSS2 abundance can boost immune infiltration for LUAD.

\section{Discussion}

As a pivotal molecule in the regulation of SARS-CoV-2 invading human host cells, TMPRSS2 is attracting massive attention in the current SARS-CoV-2 pandemic. ${ }^{32-34}$ Because SARS-CoV-2 has and is infecting large numbers of people, including many cancer patients, an investigation into the role of TMPRSS2 in cancer may provide valuable advice for treating cancer patients infected with SARS-CoV-2. Previous 
studies of TMPRSS2 in cancer mainly focused on its oncogenic role in prostate cancer. ${ }^{6-8}$ In this study, we focused on LUAD, considering that it is the most common histological type in lung cancer and that the lungs are the primary organ SARS-CoV-2 attacks. In contrast to its oncogenic role in prostate cancer, TMPRSS2 plays a tumor suppressive role in LUAD, as we have provided abundant evidence. First, TMPRSS2 downregulation correlates with elevated activities of many oncogenic pathways in LUAD, including cell cycle, mismatch repair, p53, and ECM signaling. Second, TMPRSS2 downregulation correlates with increased tumor cell proliferation, stemness, genomic instability, and ITH in LUAD. Finally, TMPRSS2 downregulation is associated with tumor advancement and worse survival in LUAD.

Furthermore, both in vitro and in vivo experiments demonstrated that TMPRSS2 downregulation markedly promoted the proliferation and invasion capacity of LUAD cells, supporting the tumor suppressor role of TMPRSS2 in LUAD.

Our bioinformatics analysis revealed significant negative associations between TMPRSS2 expression and immune signatures, including both immune-stimulatory and immune-inhibitory signatures, in LUAD (Figure 1a). Nevertheless, TMPRSS2 expression tended to have a stronger negative correlation with immune-inhibitory signatures than with immune-stimulatory signatures in LUAD (Figure 1b). The significant different levels of correlations of immune-stimulatory and immune-inhibitory signatures with TMPRSS2 expression could be a factor responsible for the worse prognosis in LUAD patients with TMPRSS2 deficiency. Furthermore, the associations between TMPRSS2 and tumor immunity in LUAD were completely verified by both in vitro and in vivo experiments. That is, knockdown of TMPRSS2 significantly increased tumor immunogenicity and immune cell infiltration in LUAD. On the other hand, both computational and experimental data showed that TMPRSS2 downregulation significantly enhanced PD-L1 expression in LUAD. Because both inflamed tumor microenvironment and PD-L1 expression are determinants of cancer response to immunotherapy, ${ }^{35}$ TMPRSS2-depleted LUAD would respond better to immunotherapy than TMPRSS2-wildtype LUAD. This was supported by our in vivo experiments showing that TMPRSS2-knockdown tumors were more sensitive to the PD-1/PD-L1 inhibitor. Thus, TMPRSS2 downregulation is a positive biomarker of immunotherapy for LUAD. In addition, because TMPRSS2 downregulation often occurs in advanced LUAD, it indicates that advanced LUAD could benefit more from immunotherapy than early-stage LUAD.

TMPRSS 2 inhibition has been indicated as a strategy for preventing and treating SARS-CoV-2 infection for the crucial role of TMPRSS2 in the SARS-CoV-2 invasion. ${ }^{33,36}$ However, our data suggest that this strategy may not be a good option for lung cancer patients in terms of the tumor suppressor role of TMPRSS2 in LUAD. Interestingly, we found that TMPRSS2 displayed significantly higher expression levels in non-smoker than in smoker LUAD patients in four LUAD cohorts in which related data were available (Student's $t$ test, $p<0.05, \mathrm{FC}>1.5$ ) (Figure 6a). This result indicates that non-smoker LUAD patients could be more susceptible to SARS-CoV-2 infection than smoker LUAD patients. As expected, non-smoker LUAD patients had significantly lower TMB and antitumor immunity than smoker LUAD patients (Figure 6b), consistent with findings from previous studies. ${ }^{37,38}$ 
In conclusion, TMPRSS2 is a tumor suppressor in LUAD, as evidenced by its downregulation correlated with increased genomic instability and ITH, tumor progression, and unfavorable clinical outcomes in LUAD. However, TMPRSS2 downregulation is a positive biomarker of immunotherapy for LUAD. Our data provide implications in the connection between lung cancer and pneumonia caused by SARS-CoV-2 infection.

\section{Abbreviations}

ACE2: angiotensin-converting enzyme 2; CCK-8: the Cell Counting Kit-8; DAPI: 4',6- diamidino-2phenylindole; DDR: DNA damage repair; DFS: disease-free survival; ECM: extracellular matrix; FC: fold change; FDR: false discovery rate; GO: gene ontology; GSEA: gene set enrichment analysis; HRD: Homologous recombination deficiency; ICls: immune checkpoint inhibitors; ITH: intratumor heterogeneity; LUAD: lung adenocarcinoma; LUSC: lung squamous cell carcinoma; MDSCs: myeloidderived suppressor cells; OS: overall survival; PI: proximal-inflammatory; PP: proximal-proliferative; RTPCR: Real-Time PCR; S: spike glycoprotein; SARS-CoV-2: severe acute respiratory syndrome coronavirus 2; siRNA: small interfering RNA; ssGSEA: single-sample gene-set enrichment analysis; TCGA: The Cancer Genome Atlas; TILs: tumor-infiltrating lymphocytes; TMB: tumor mutation burden; TMPRSS2: transmembrane protease serine 2; TRU: terminal respiratory unit; WGCNA: weighted gene co-expression network analysis

\section{Declarations}

\section{Ethics approval and consent to participate}

The study was done in accordance with both the Declaration of Helsinki and the International Conference on Harmonization Good Clinical Practice guidelines and was approved by the institutional review board.

\section{Consent for publication}

Not applicable.

\section{Availability of data and material}

The five LUAD genomic datasets were obtained from the Genomic Data Commons Data Portal (https://portal.gdc.cancer.gov/) and the Gene Expression Omnibus (https://www.ncbi.nlm.nih.gov/geo/).

\section{Competing Interests}

The authors declare that they have no competing interests. 


\section{Funding}

This work was supported by the China Pharmaceutical University (grant number 3150120001 to XW), Natural Science Foundation of Jiangsu Province冈grant number BK20201090 to ZL), and China Postdoctoral Science Foundation (grant number 2021M691338 to ZL)

\section{Authors' contributions}

Zhixian Liu: Validation, Formal analysis, Resources, Investigation, Data curation, Visualization, Writing original draft, Funding acquisition. Zhilan Zhang: Software, Formal analysis, Investigation, Data curation, Visualization. Qiushi Feng: Software, Formal analysis, Visualization. Xiaosheng Wang: Conceptualization, Methodology, Resources, Investigation, Writing - original draft, Writing - review \& editing, Supervision, Project administration, Funding acquisition.

\section{Acknowledgements}

\section{References}

1. Ou X, et al. Characterization of spike glycoprotein of SARS-CoV-2 on virus entry and its immune cross-reactivity with SARS-CoV. Nat Commun. 2020;11:1620. doi:10.1038/s41467-020-15562-9.

2. Tomlins SA, et al. Role of the TMPRSS2-ERG gene fusion in prostate cancer. Neoplasia. 2008;10:177-88. doi:10.1593/neo.07822.

3. Katopodis $\mathrm{P}$, et al. Pan-cancer analysis of transmembrane protease serine 2 and cathepsin $L$ that mediate cellular SARS-CoV-2 infection leading to COVID-19. Int J Oncol. 2020;57:533-9. doi:10.3892/ijo.2020.5071.

4. Kong Q, et al. Analysis of the susceptibility of lung cancer patients to SARS-CoV-2 infection. Mol Cancer. 2020;19:80. doi:10.1186/s12943-020-01209-2.

5. Bao R, Hernandez K, Huang L, Luke JJ. ACE2 and TMPRSS2 expression by clinical, HLA, immune, and microbial correlates across 34 human cancers and matched normal tissues: implications for SARS-CoV-2 COVID-19. J Immunother Cancer 8, doi:10.1136/jitc-2020-001020 (2020).

6. Mollica V, Rizzo A, Massari F. The pivotal role of TMPRSS2 in coronavirus disease 2019 and prostate cancer. Future Oncol. 2020;16:2029-33. doi:10.2217/fon-2020-0571.

7. Trifunovski A, et al. Detection of TMPRSS2-ERG Fusion Transcript in Biopsy Specimen of Prostate Cancer Patients: A Single Centre Experience. Pril (Makedon Akad Nauk Umet Odd Med Nauki). 2020;41:5-14. doi:10.2478/prilozi-2020-0018.

8. Dudka I, et al. Comprehensive metabolomics analysis of prostate cancer tissue in relation to tumor aggressiveness and TMPRSS2-ERG fusion status. BMC Cancer. 2020;20:437. doi:10.1186/s12885020-06908-z. 
9. Luo L, et al. TMPRSS2 Correlated With Immune Infiltration Serves as a Prognostic Biomarker in Prostatic Adenocarcinoma: Implication for the COVID-2019. Front Genet. 2020;11:575770. doi:10.3389/fgene.2020.575770.

10. Ding $L$, et al. Somatic mutations affect key pathways in lung adenocarcinoma. Nature. 2008;455:1069-75. doi:10.1038/nature07423.

11. Rousseaux $S$, et al. Ectopic activation of germline and placental genes identifies aggressive metastasis-prone lung cancers. Sci Transl Med. 2013;5:186ra166. doi:10.1126/scitranslmed.3005723.

12. Okayama $\mathrm{H}$, et al. Identification of genes upregulated in ALK-positive and EGFR/KRAS/ALK-negative lung adenocarcinomas. Cancer Res. 2012;72:100-11. doi:10.1158/0008-5472.Can-11-1403.

13. Der SD, et al. Validation of a histology-independent prognostic gene signature for early-stage, nonsmall-cell lung cancer including stage IA patients. J Thorac Oncol. 2014;9:59-64. doi:10.1097/jto.0000000000000042.

14. Hänzelmann S, Castelo R, Guinney J. GSVA: gene set variation analysis for microarray and RNA-seq data. BMC Bioinformatics. 2013;14:7. doi:10.1186/1471-2105-14-7.

15. Subramanian A, et al. Gene set enrichment analysis: a knowledge-based approach for interpreting genome-wide expression profiles. Proc Natl Acad Sci U S A. 2005;102:15545-50. doi:10.1073/pnas.0506580102.

16. Kanehisa M, Furumichi M, Tanabe M, Sato Y, Morishima K. KEGG: new perspectives on genomes, pathways, diseases and drugs. Nucleic Acids Res. 2017;45:D353-d361. doi:10.1093/nar/gkw1092.

17. Langfelder P, Horvath S. WGCNA: an R package for weighted correlation network analysis. BMC Bioinformatics. 2008;9:559. doi:10.1186/1471-2105-9-559.

18. Takahashi $\mathrm{H}$, et al. Immune Cytolytic Activity for Comprehensive Understanding of Immune Landscape in Hepatocellular Carcinoma. Cancers (Basel) 12, doi:10.3390/cancers12051221 (2020).

19. Miranda A, et al. Cancer stemness, intratumoral heterogeneity, and immune response across cancers. Proc Natl Acad Sci U S A. 2019;116:9020-9. doi:10.1073/pnas.1818210116.

20. Li WY, et al. The role of EGFR mutation as a prognostic factor in survival after diagnosis of brain metastasis in non-small cell lung cancer: a systematic review and meta-analysis. BMC Cancer. 2019;19:145. doi:10.1186/s12885-019-5331-z.

21. Comprehensive molecular profiling of lung adenocarcinoma. Nature. 2014;511:543-50. doi:10.1038/nature13385.

22. Ferguson LR, et al. Genomic instability in human cancer: Molecular insights and opportunities for therapeutic attack and prevention through diet and nutrition. Semin Cancer Biol 35 Suppl, S5-s24, doi:10.1016/j.semcancer.2015.03.005 (2015).

23. Palmieri G, et al. Genetic instability and increased mutational load: which diagnostic tool best direct patients with cancer to immunotherapy? J Transl Med. 2017;15:17. doi:10.1186/s12967-017-1119-6. 
24. Smith JC, Sheltzer JM. Systematic identification of mutations and copy number alterations associated with cancer patient prognosis. Elife 7, doi:10.7554/eLife.39217 (2018).

25. Knijnenburg TA, et al. Genomic and Molecular Landscape of DNA Damage Repair Deficiency across The Cancer Genome Atlas. Cell Rep. 2018;23:239-54.e236. doi:10.1016/j.celrep.2018.03.076.

26. McKinney JA, et al. Distinct DNA repair pathways cause genomic instability at alternative DNA structures. Nat Commun. 2020;11:236. doi:10.1038/s41467-019-13878-9.

27. Eischen CM. Genome Stability Requires p53. Cold Spring Harb Perspect Med 6, doi:10.1101/cshperspect.a026096 (2016).

28. Burrell RA, McGranahan N, Bartek J, Swanton C. The causes and consequences of genetic heterogeneity in cancer evolution. Nature. 2013;501:338-45. doi:10.1038/nature12625.

29. Li M, Zhang Z, Li L, Wang X. An algorithm to quantify intratumor heterogeneity based on alterations of gene expression profiles. Commun Biol. 2020;3:505. doi:10.1038/s42003-020-01230-7.

30. Walker C, Mojares E. \& Del Río Hernández, A. Role of Extracellular Matrix in Development and Cancer Progression. Int J Mol Sci 19, doi:10.3390/ijms19103028 (2018).

31. Patel SP, Kurzrock R. PD-L1 Expression as a Predictive Biomarker in Cancer Immunotherapy. Mol Cancer Ther. 2015;14:847-56. doi:10.1158/1535-7163.Mct-14-0983.

32. Zang R, et al. TMPRSS2 and TMPRSS4 promote SARS-CoV-2 infection of human small intestinal enterocytes. Sci Immunol 5, doi:10.1126/sciimmunol.abc3582 (2020).

33. Hoffmann M, et al. SARS-CoV-2 Cell Entry Depends on ACE2 and TMPRSS2 and Is Blocked by a Clinically Proven Protease Inhibitor. Cell. 2020;181:271-80.e278. doi:10.1016/j.cell.2020.02.052.

34. Bestle D, et al. TMPRSS2 and furin are both essential for proteolytic activation of SARS-CoV-2 in human airway cells. Life Sci Alliance 3, doi:10.26508/Isa.202000786 (2020).

35. Cristescu R, et al. Pan-tumor genomic biomarkers for PD-1 checkpoint blockade-based immunotherapy. Science 362, doi:10.1126/science.aar3593 (2018).

36. Stopsack KH, Mucci LA, Antonarakis ES, Nelson PS, Kantoff PW. TMPRSS2 and COVID-19: Serendipity or Opportunity for Intervention? Cancer Discov. 2020;10:779-82. doi:10.1158/21598290.Cd-20-0451.

37. Chae YK, et al. Association of Tumor Mutational Burden With DNA Repair Mutations and Response to Anti-PD-1/PD-L1 Therapy in Non-Small-Cell Lung Cancer. Clin Lung Cancer. 2019;20:88-96.e86. doi:10.1016/j.cllc.2018.09.008.

38. Kinoshita $\mathrm{T}$, et al. Determination of poor prognostic immune features of tumour microenvironment in non-smoking patients with lung adenocarcinoma. Eur J Cancer. 2017;86:15-27. doi:10.1016/j.ejca.2017.08.026.

\section{Figures}


Figure 1

a

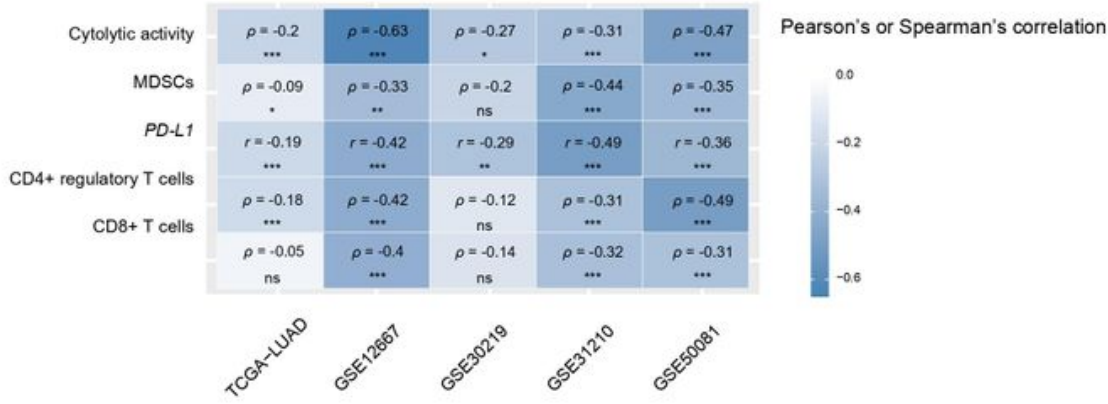

b
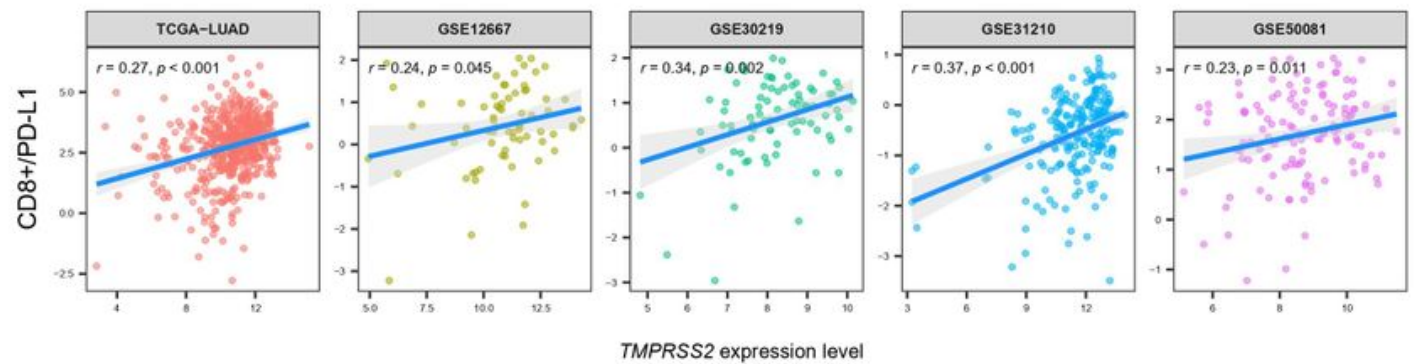

c

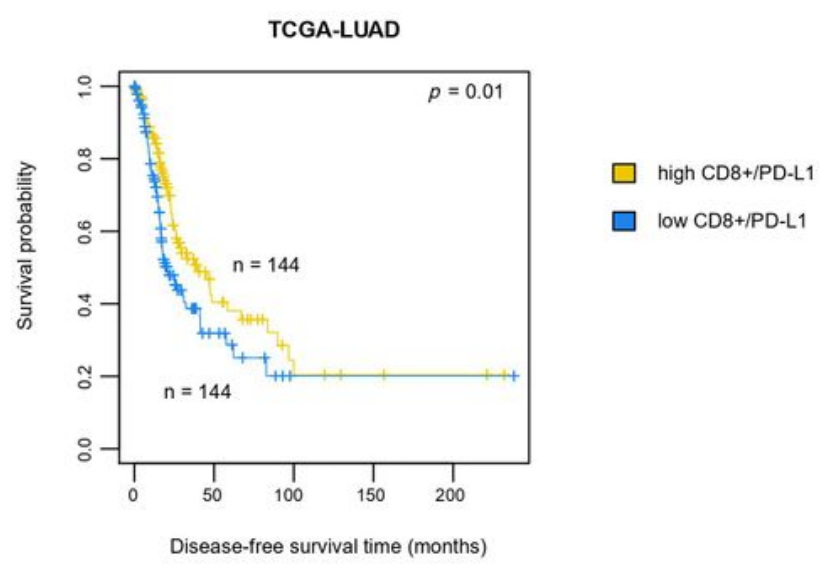

Figure 1

Association between TMPRSS2 expression and immune signatures in LUAD. (a) Correlations between TMPRSS2 expression levels and the enrichment levels of CD8+ T cells and NK cells, immune cytolytic activity, $P D-L 1$ expression levels, and the enrichment levels of CD4+ regulatory T cells and MDSCs in five LUAD cohorts. The Spearman or Pearson correlation coefficients ( $\rho$ or $r$ ) and $p$ values are shown. (b) Pearson correlations between TMPRSS2 expression levels and the ratios of immune-stimulatory/immune- 
inhibitory signatures (CD8+/PD-L1) in LUAD. (c) Kaplan-Meier survival curves showing a better diseasefree survival in LUAD patients with high ratios of CD8+/PD-L1 (upper third) than those with low ratios of CD8+/PD-L1 (bottom third). The log-rank test $p$ value is shown. ${ }^{*} p<0.05,{ }^{*} p<0.01,{ }^{\star * \star} p<0.001,{ }^{\text {ns }} p \geq$ 0.05. They also apply to the following figures.

Figue 2
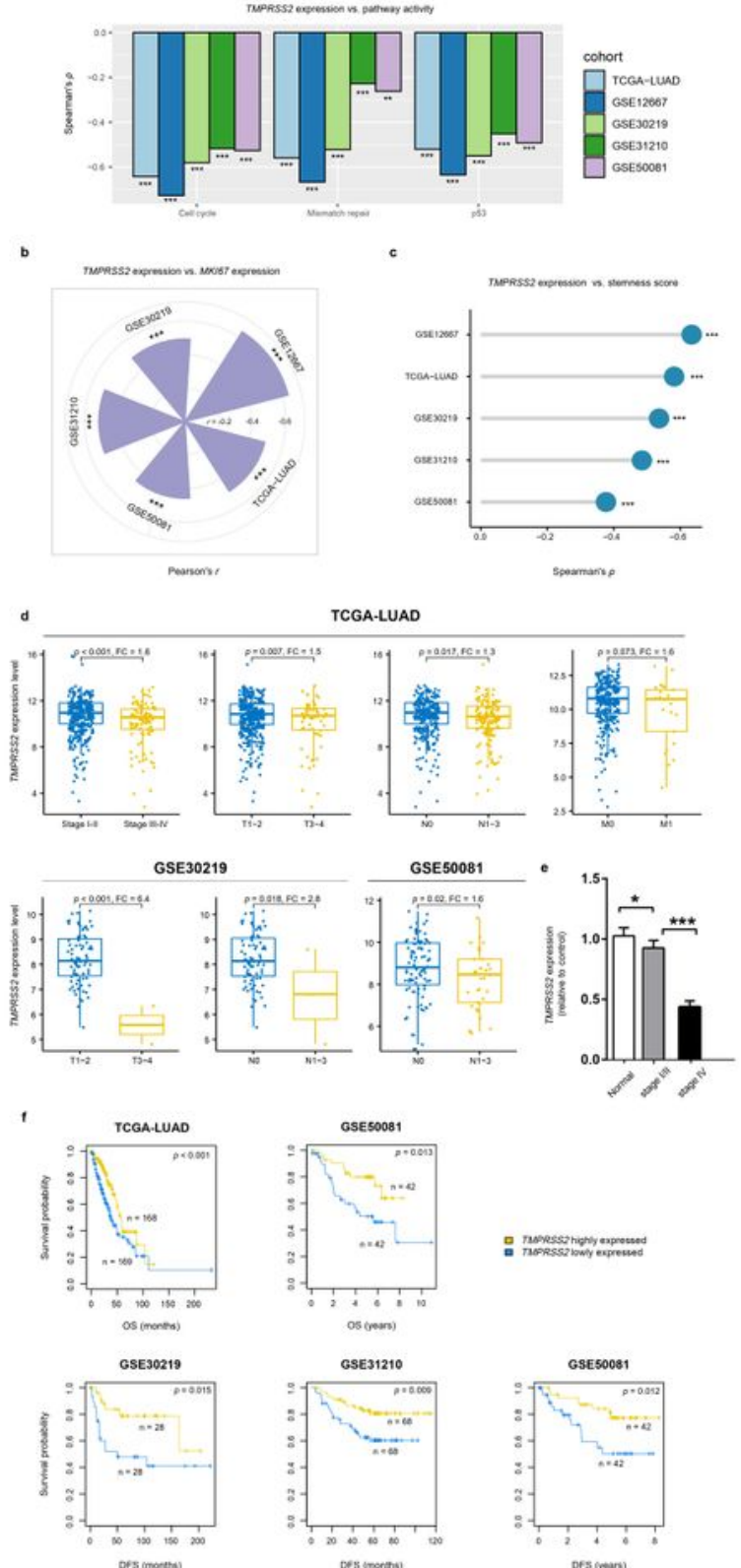

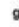
TCGA.LUAD
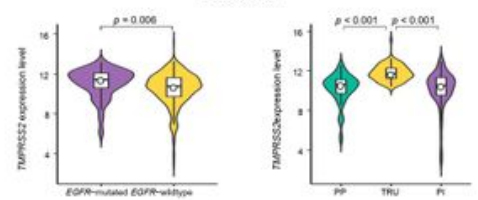

Figure 2 
Associations between TMPRSS2 expression and oncogenic pathways, tumor phenotypes and prognosis in LUAD. The inverse correlations between TMPRSS2 expression levels and the activities of oncogenic pathways (a), KI67 expression levels (b), and stemness scores (c) in LUAD. The Spearman or Pearson correlation coefficients ( $\rho$ or $r$ ) and $p$ values are shown. (d) Comparisons of TMPRSS2 expression levels between late-stage (Stage III-IV) and early-stage (Stage I-II), between large-size (T3-4) and small-size (T12), and between N1-3 (lymph nodes) and N0 (without regional lymph nodes) LUADs. The Student's $t$ test $p$ values and fold change (FC) of mean TMPRSS2 expression levels are shown. (e) The lung cancer data from Jiangsu Cancer Hospital showing that TMPRSS2 expression levels are significantly lower in latestage (Stage IV) than in early-stage (Stage I-II) LUADs. (f) Kaplan-Meier survival curves showing that lowTMPRSS2-expression-level (bottom third) LUAD patients have worse OS and/or DFS than high-TMPRSS2expression-level (upper third) LUAD patients. The log-rank test $p$ values are shown. OS, overall survival. DFS, disease-free survival. (g) Comparisons of TMPRSS2 expression levels between EGFR-mutated and EGFR-wildtype LUADs and between three LUAD transcriptional subtypes. TRU, terminal respiratory unit. $\mathrm{PI}$, proximal-inflammatory. PP, proximal-proliferative. 

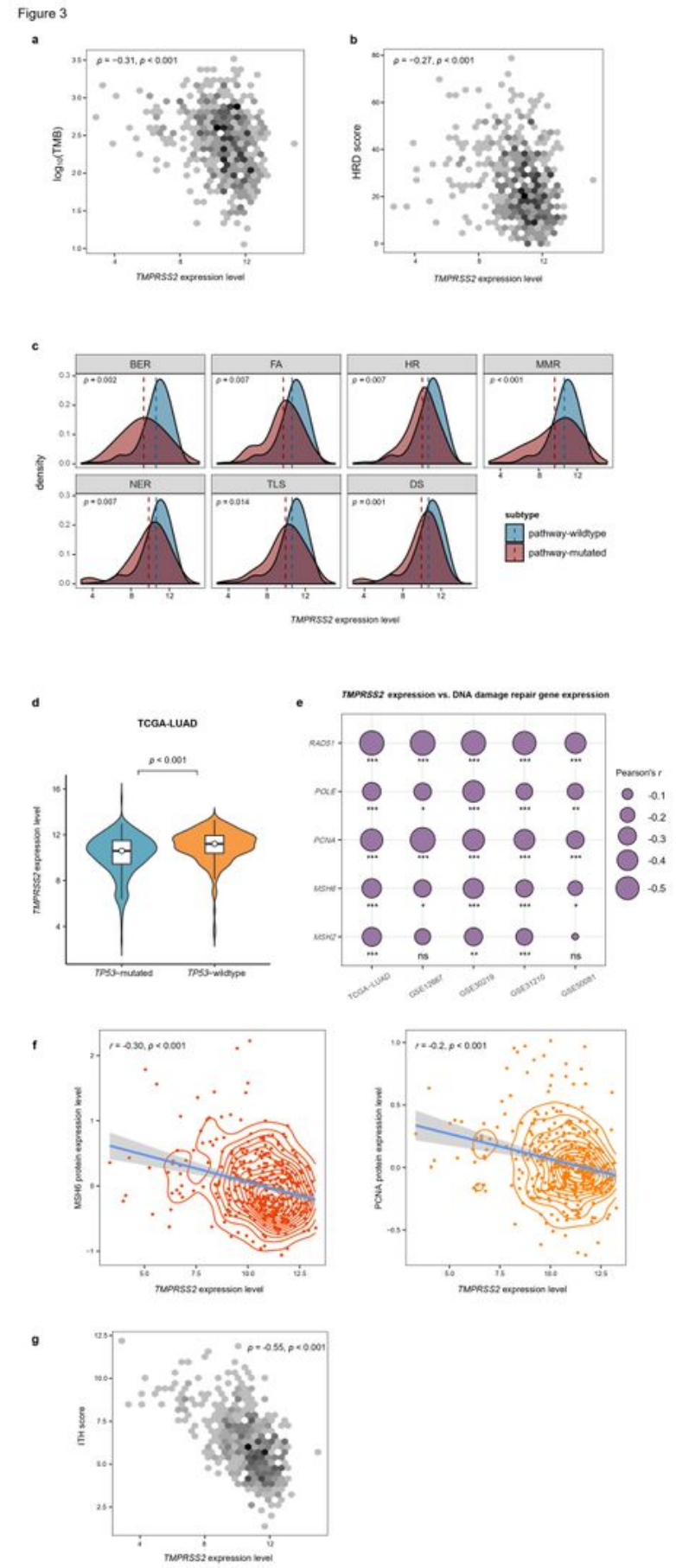

\section{Figure 3}

Association between TMPRSS2 expression and genomic instability in LUAD. Spearman correlations between TMPRSS2 expression levels and tumor mutation burden (TMB) (a) and homologous recombination deficiency (HRD) scores (b) in TCGA-LUAD. TMB is the total somatic mutation count in the tumor. The HRD scores were obtained from the publication. ${ }^{25}$ (c) Comparisons of TMPRSS2 expression levels between pathway-wildtype and pathway-mutated LUAD subtypes for seven DNA damage repair 
(DDR) pathways in TCGA-LUAD. The pathway-wildtype indicates no deleterious mutations in any pathway genes, and the pathway-mutated indicates at least a deleterious mutation in pathway genes. BER, base excision repair. FA, Fanconi anemia. HR, homologous recombination. MMR, mismatch repair. NER, nucleotide excision repair. TLS, translesion DNA synthesis. DS, damage sensor. (d) Comparisons of TMPRSS2 expression levels between TP53-mutated and TP53-wildtype LUADs. Expression correlations between TMPRSS2 and DDR-associated genes (e) and proteins (f) in LUAD. (g) Spearman correlation between TMPRSS2 expression levels and intratumor heterogeneity (ITH) scores. The ITH scores were evaluated by the DEPTH algorithm. ${ }^{29}$ 
Figure 4

a
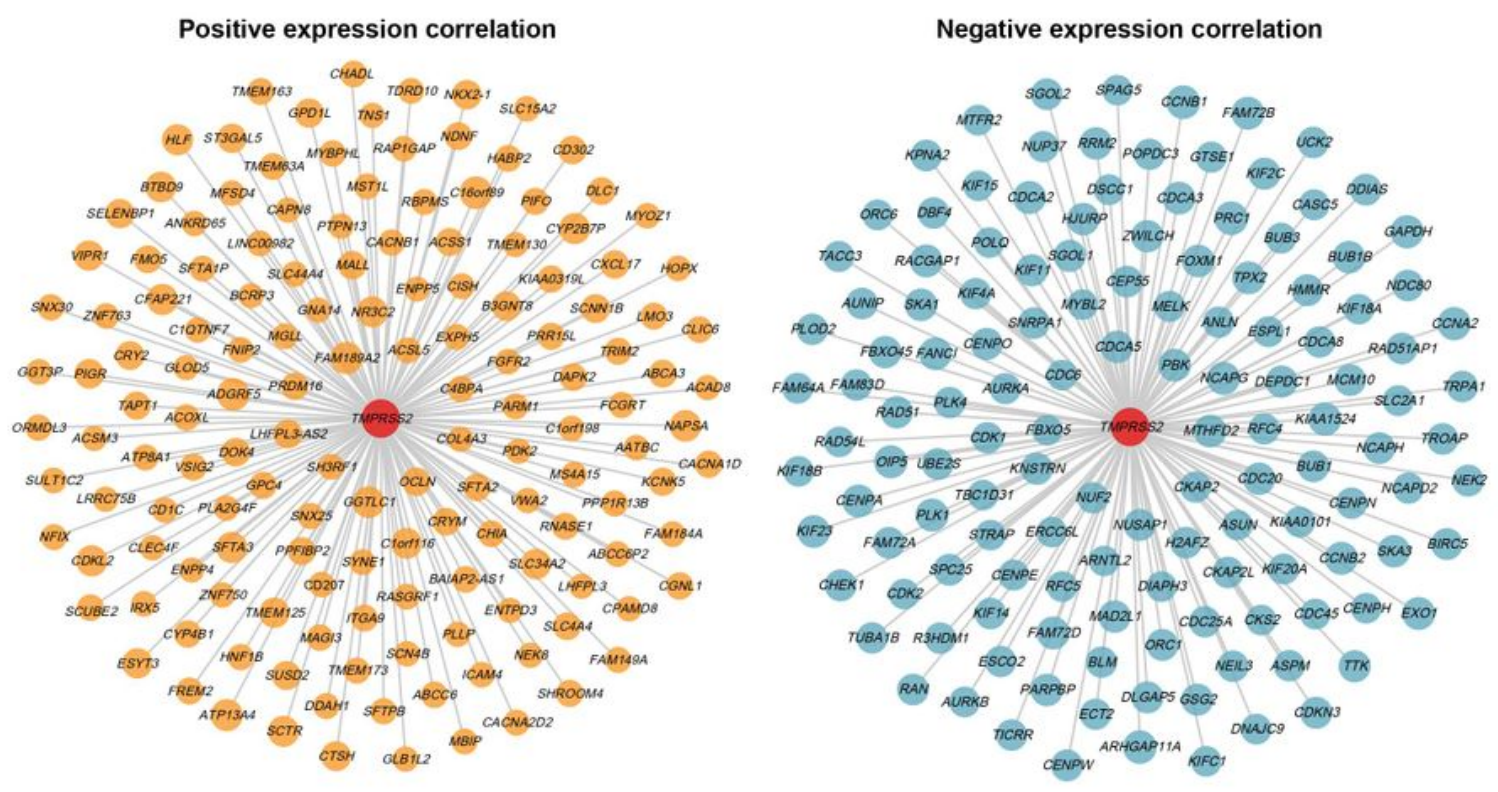

b

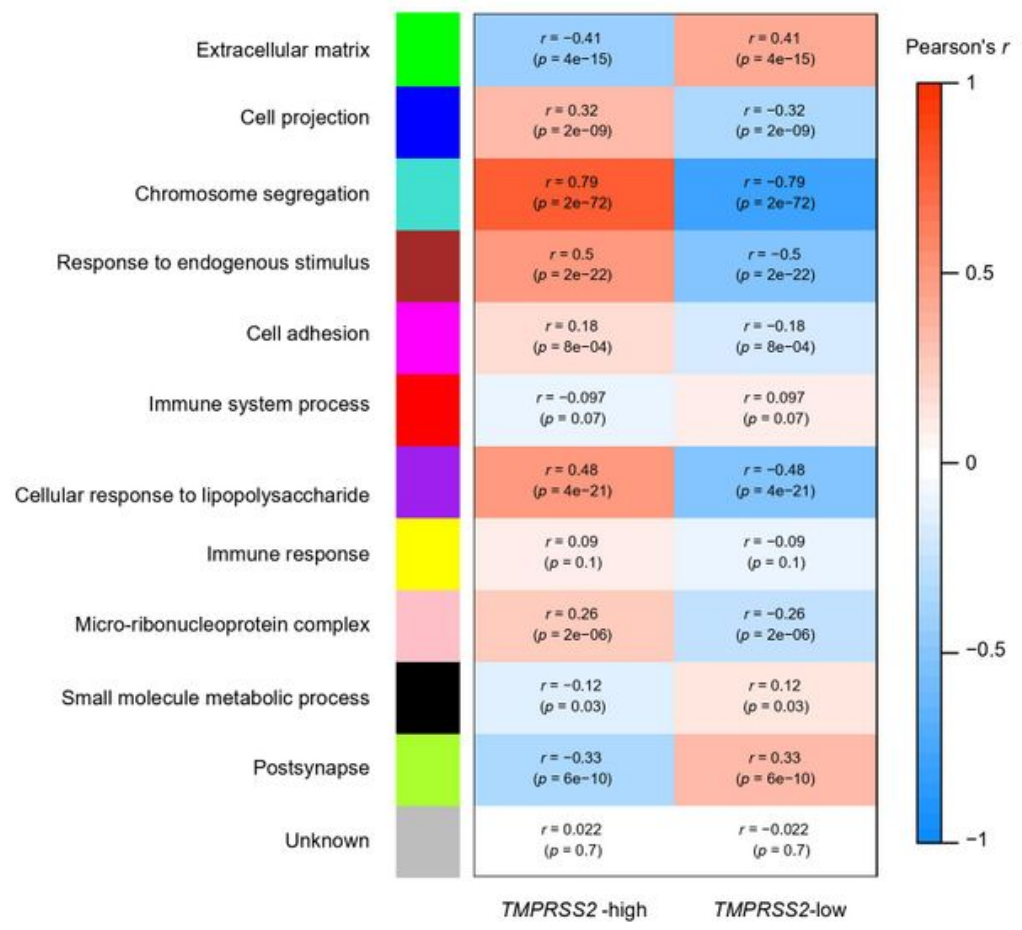

Figure 4

Co-expression networks of TMPRSS2 in LUAD. (a) 150 and 135 genes having strong positive and negative expression correlations with TMPRSS2 in TCGA-LUAD, respectively $(|| \mid>0.5)$. (b) Gene modules and their representative gene ontology terms highly enriched in high- (upper third) and low-TMPRSS2expression-level (bottom third) LUADs identified by WGCNA. ${ }^{17}$ 
Figure 5(a-f)

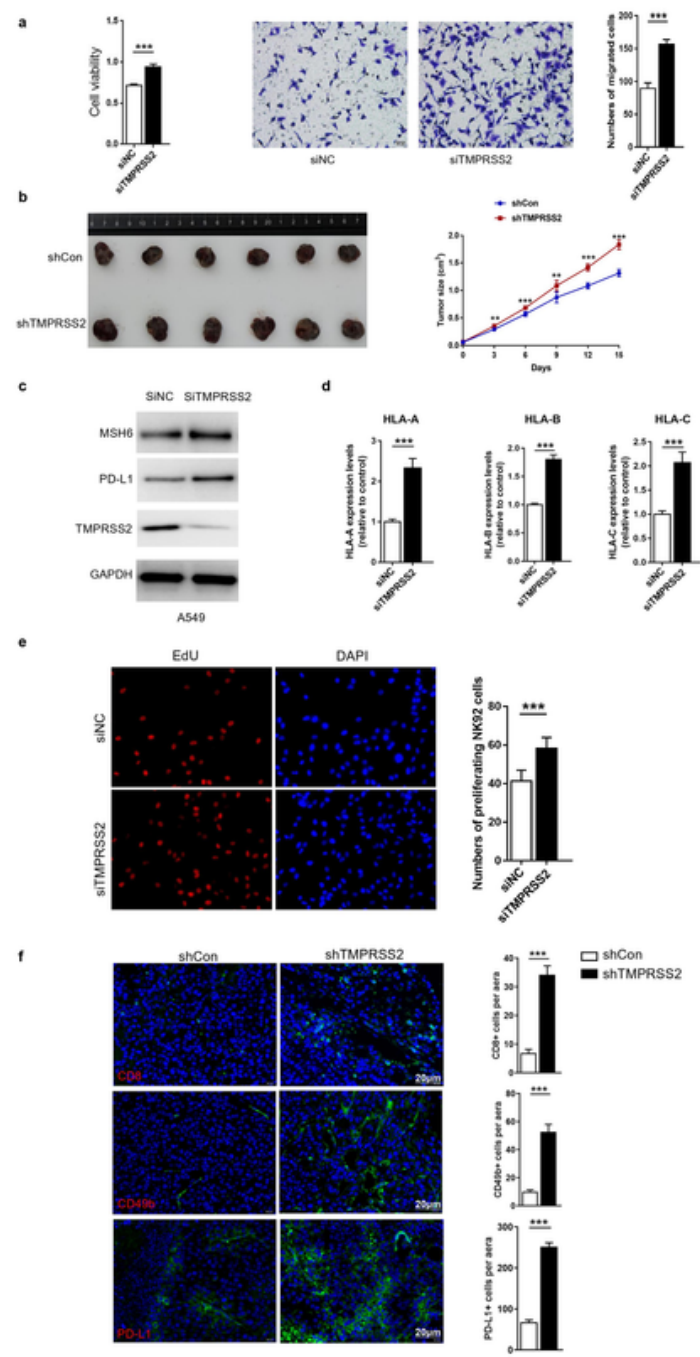

Figure $5(g-m)$
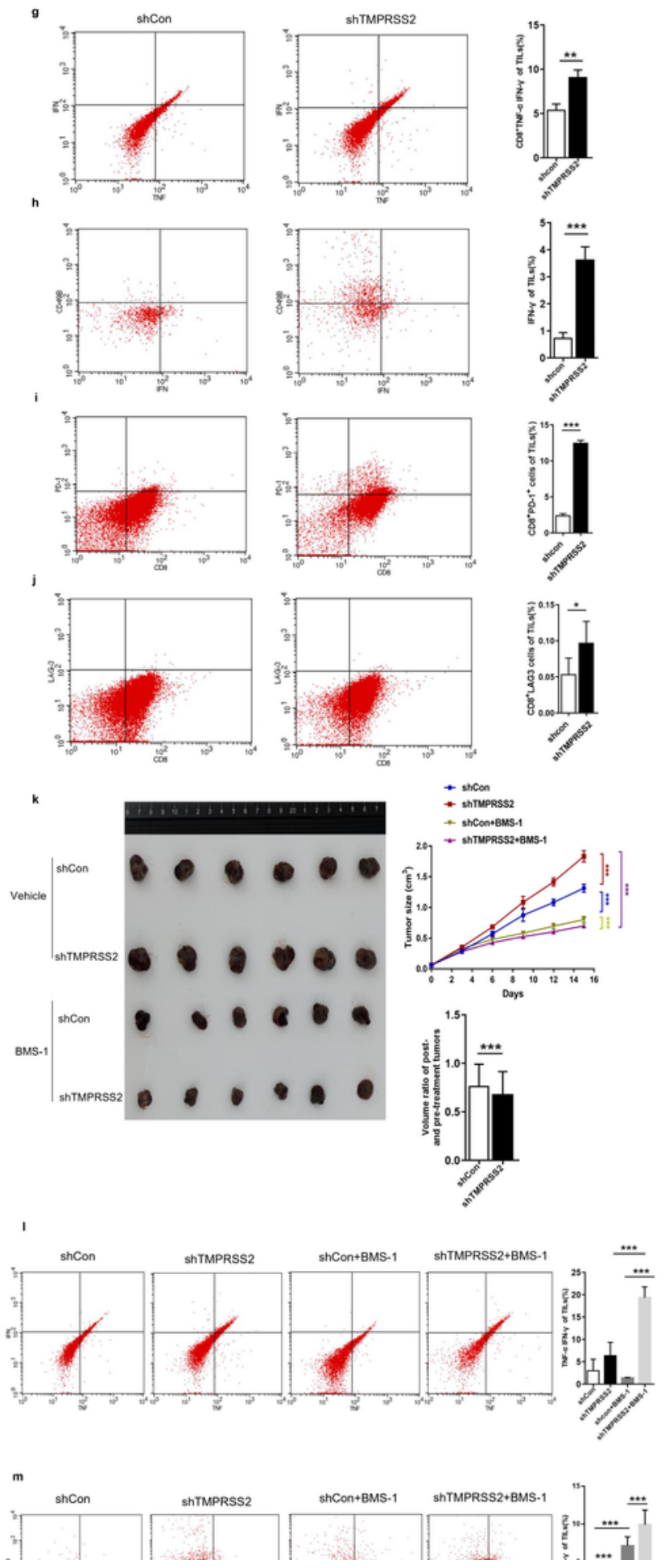

\section{Figure 5}

In vivo and in vitro experimental validation of the bioinformatics findings. TMPRSS2-knockdown tumors display increased tumor-infiltrating lymphocytes, expression of immune checkpoint molecules, and sensitization to immune checkpoint inhibitors. (a) TMPRSS2 knockdown markedly promoted proliferative 
and invasive abilities of A549 cells. (b) TMPRSS2 knockdown increased tumor volume and progression in Lewis tumor mouse models. Lewis tumor cells transfected with ShCon or ShTMPRSS2 lentivirus were subcutaneously injected into mice. The tumor volumes were measured every three days from the fifth day to the fifteenth. Data represent mean \pm SEM. SEM, standard error of mean. ShTMPRSS2 versus ShCon group, $n=6$ for each group, two-tailed Student's $t$ test, $* p<0.05$, $* * p<0.01$, $* \star * p<0.001$. (c) TMPRSS2 knockdown increased MSH6 expression in A549 cells, as evidenced by Western blotting. (d) TMPRSS2 knockdown enhanced the expression of MHC class I genes ( $H L A-A, H L A-B$, and $H L A-C$ ) in A549 cells, as evidenced by real-time qPCR. (e) NK cells co-cultured with TMPRSS2-knockdown A549 cells showing higher proliferation capacity than NK cells co-cultured with TMPRSS2-wildtype A549 cells, as evidenced by the EDU proliferation assay. (f) CD8, CD49b, and PD-L1 immunofluorescence staining in Lewis orthotopic tumors and H-score analysis. ShTMPRSS2 versus shCon group, $n=6$ for each group, twotailed Student's $t$ test, $* * * p<0.001$. (g-j) Comparisons of TNF-a, IFN- $y$, PD-1, and LAG3 expression on CD8+ T cells from tumor-infiltrating lymphocytes (TILs) in tumor-bearing mice between TMPRSS2knockdown and TMPRSS2-wildtype group (ShTMPRSS2 versus ShCon group, $n=6$ for each group, twotailed Student's $t$ test, ${ }^{*} p<0.05$, $* \star p<0.01$, ${ }^{\star * \star} p<0.001$ ). TILs were stained with CD3, CD8, TNF-a, and IFN- $y$ and were then analyzed by flow cytometry. Lymphocytes were gated according to forward scatter and side scatter. CD3 and CD8 staining was used to identify CD8+ T cells. (k-m) TMPRSS2-knockdown tumors formed by subcutaneous injection of Lewis cells, as mentioned in (b). shCon and shTMPRSS2 tumor-bearing mice were divided into vehicle and BMS-1 groups. The vehicle and BMS-1 groups of mice were treated with solvent and BMS-1, respectively. (k) Representative images of tumor-bearing mice shown on the left. The right graph showing the change of tumor size in the tumor-bearing mice over time. Data represent mean \pm SEM ( $n=6$ for each group, two-tailed Student's $t$ test, ${ }^{\star} p<0.05$, ${ }^{\star \star} p<0.01$, $\star \star \star p<0.001)$; Comparison of the volume ratios of mice tumors after and before treatment with BMS-1 between TMPRSS2-knockdown and TMPRSS2-wildtype groups (two-tailed Student's $t$ test, ${ }^{\star \star \star} p<0.001$ ). Comparisons of TNF- $a(\mathbf{I})$ and IFN- $y(\mathbf{m})$ expression on CD8+ T cells from TILs in tumor-bearing mice $(n=$

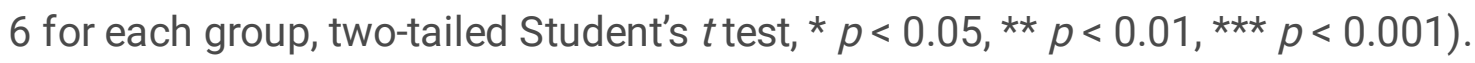


Figure 6

a
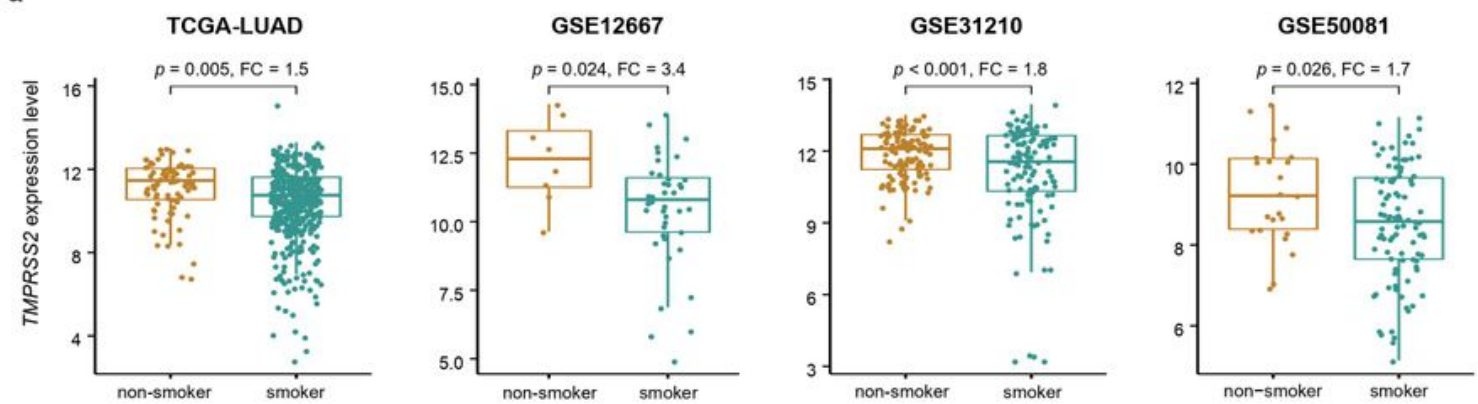

b
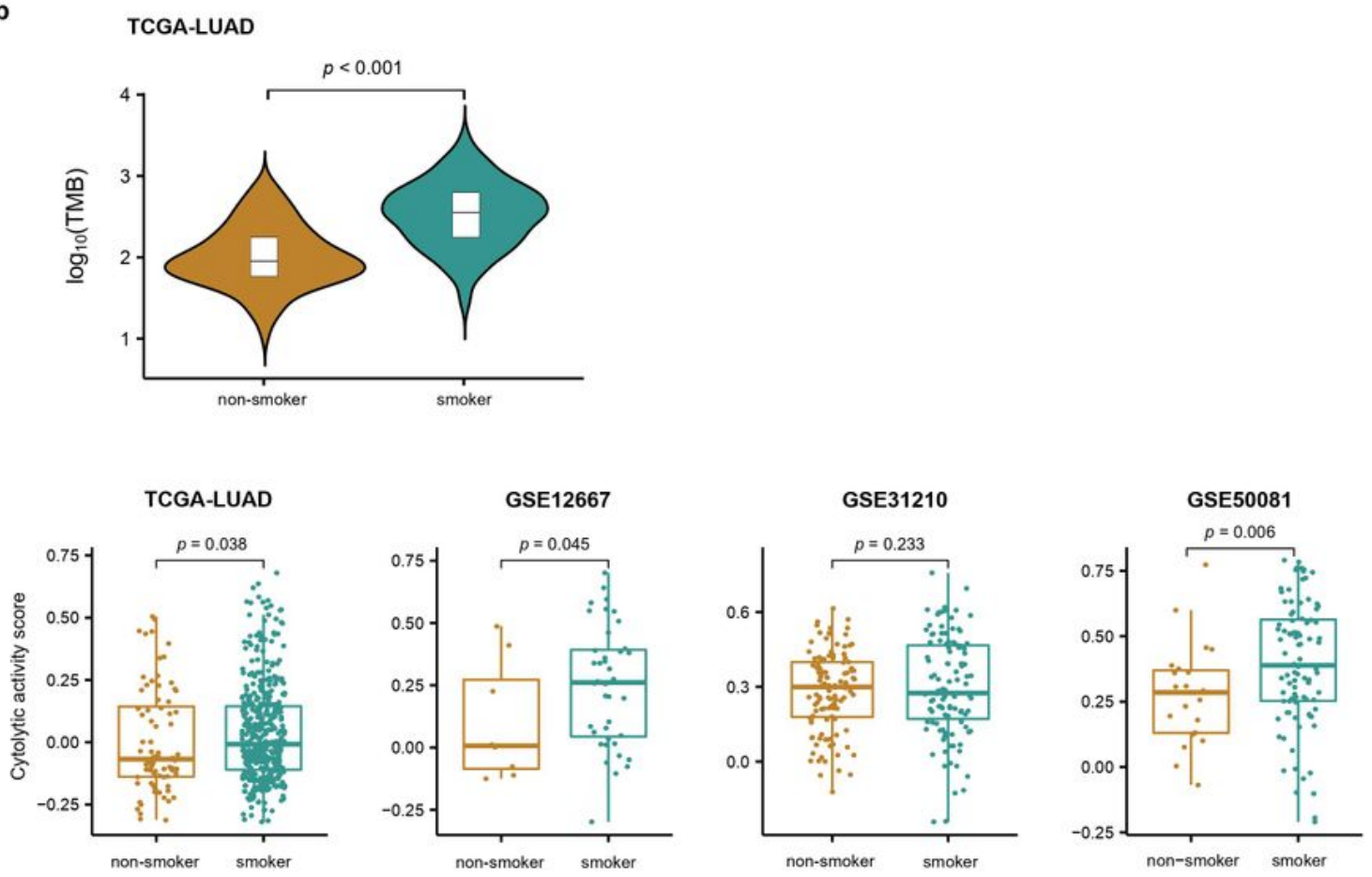

Figure 6

Comparisons of TMPRSS2 expression levels, TMB, and immune signatures between non-smoker and smoker LUADs. Non-smoker LUAD patients showing significantly higher TMPRSS2 expression levels (a) and lower TMB and immune signature scores (b) than smoker LUAD patients. The two-tailed Student's $t$ test and one-tailed Mann-Whitney Utest $p$ values are shown in (a) and (b), respectively. 


\section{Supplementary Files}

This is a list of supplementary files associated with this preprint. Click to download.

- Supplementarytables.xlsx 